\title{
On the relationship between limiting current and mass-transport impedance at zero frequency
}

David A. Harrington ${ }^{1}$

Chemistry Department, University of Victoria, Victoria, BC, V8W 2Y2, Canada)

The zero-frequency reversible mass transport impedance (measured at the reversible potential) is simply related to the limiting current density (far from the reversible potential). For mass transport of a single species exchanging $n$ electrons with the electrode, their product is $R T / n F$. The conditions under which this relationship holds are investigated, and it is shown that it applies for most of the mass-transport situations found in electrochemistry, including with forced convection or for electrodes without uniform accessibility. For the two-species case, $\mathrm{O}+n \mathrm{e}^{-} \rightleftharpoons \mathrm{R}$, the same product is found, provided the harmonic mean of the anodic and cathodic limiting currents is used.

a) Electronic mail: dharr@uvic.ca 
Electrochemical impedance spectroscopy is increasingly used as a tool in cell geometries with complicated convective diffusion mass transport, such as in microfluidic devices. Fast redox couples are still widely used as test systems, and understanding the mass-transport impedance in these situations is important. Analytical solution of the convective diffusion equations is often difficult, especially in cases where uniform accessibility cannot be assumed, and finite-element numerical solutions are frequently used. Knowing theoretically where the zero-frequency impedance is can be useful for extrapolation purposes or for determining if low enough frequencies have been measured. The relationship between the slope of the steady-state polarization curve and the zero-frequency intercept of the impedance at the same potential is well known ${ }^{1,2}$. Bard and Faulkner's textbook also discusses a relationship between the mass-transport resistance and the limiting current for reversible systems ${ }^{3}$. The mass transport resistance there is equal to the zero-frequency intercept of impedance, and its product with the limiting current is $R T / n F$. The treatment there assumes the Nernst layer approximation is valid, and does not explicitly consider convection. Here the exact conditions for this relationship to hold are investigated. Although the object of interest here is the reversible mass transport impedance, the single-species version is usually considered as "the" mass transport impedance and used as a building block in coupling to more complicated $\operatorname{kinetics}^{4,5}$.

The relationship here is established for all the common mass-transport conditions found in electrochemistry, including with forced convection, under the usual assumptions that apply in excess supporting electrolyte. Universal relationships across different geometries are known for some systems, e.g., pulse sequences under reversible conditions when all the diffusivities are equal ${ }^{6}$. In the case of impedance, the relationship between the governing equations for impedance and step experiments for the rotating disk electrode (RDE) may have been used to predict the zero-frequency intercept in that case ${ }^{5,7}$. Much later, Bezegh and Janata ${ }^{8}$ noted the Laplace transform relationship between the time dependence in the (single-species, 1-D) Cottrell equation and the frequency dependence of the Warburg impedance, and proposed that the latter could be derived from the former using Laplace transformation of a perturbing potential step of magnitude $K$. They argued from the known two-species form of the Warburg impedance that $K=R T / n F$. The mixing of the twospecies Warburg and single-species Cottrell equation in the same problem is opaque, and the physical significance of the deduced step-magnitude $R T / n F$ is unclear: it would not 
be sufficient to ensure the zero surface concentration required to give the Cottrell current. There is also no mention of the boundary conditions required for this relationship to hold. Nonetheless, they used this analogy to deduce an approximation to the impedance at low frequencies for inlaid circular disk electrodes, a 3-D problem. Compton and coworkers applied this method to 2-D diffusion to microband electrodes ${ }^{9,10}$ to find approximations above a critical minimum frequency.

A recent paper by Tran et al. ${ }^{11}$ discusses the relationships between the limiting current and mass transport impedance. The authors link the kinetic and limiting currents of the Koutecky-Levich equation to the charge-transfer and mass transfer impedances for the specific case of finite-length 1-D diffusion. However, they do not discuss the reversible case or more general convective diffusion mass transport conditions that are the topic of the present work.

The problem is approached here by establishing a link between the governing convectivediffusion equations and their boundary conditions for the limiting current and zero-frequency cases. In this way, the relationship between the two is derived directly, and the conditions under which it applies are made explicit.

\section{THEORETICAL}

Consider first the reversible impedance for mass-transport of a single species, say a metal cation $\mathrm{M}^{n+}$ in equilibrium with its metal. At the reversible potential there is no dc concentration gradient in solution; the dc concentration is everywhere its bulk value $c^{\mathrm{b}}$. Under the reversibility assumption, the Nernst equation always applies at the electrode surface, so that in an ac experiment, an increase in the potential leads to an immediate increase in the surface concentration of the ion, i.e., the potential and surface concentrations are in phase. In other words, oscillation of the potential leads to a proportional oscillation of the concentration, in accordance with the small signal (linearized) Nernst equation ${ }^{4}$ :

$$
\widetilde{E}=\left(R T / n F c^{\mathrm{b}}\right) \widetilde{c}_{\mathrm{s}}
$$

where the tildes denote the complex phasors in the usual way. (The subscript s denotes evaluation at the electrode surface, and for concentration derivatives will also imply the normal component.) The boundary condition at the electrode surface is therefore that $\widetilde{c}_{\mathrm{s}}$ is 
constant at a value determined by the applied potential amplitude. The measured response is the oscillating current, which is proportional to the oscillating flux at the electrode surface:

$$
\widetilde{j}=n F\left\langle\widetilde{J}_{\mathrm{s}}\right\rangle=-n F D\left\langle(\boldsymbol{\nabla} \widetilde{c})_{\mathrm{s}}\right\rangle
$$

Here the angle brackets denote the average over the surface, which may be omitted in the case of electrodes with uniform accessibility. Any convective component of the flux is assumed to be zero at the surface, in accordance with the usual no-slip boundary condition for the fluid velocity. The mass-transport impedance is

$$
Z_{\mathrm{d}}=\frac{\widetilde{E}}{\widetilde{j}}=\frac{R T}{n^{2} F^{2} D c^{\mathrm{b}}} \cdot \frac{-\widetilde{c}_{\mathrm{s}}}{\left\langle(\boldsymbol{\nabla} \widetilde{c})_{\mathrm{s}}\right\rangle}
$$

The quantity $-\widetilde{c}_{\mathrm{s}} /\left\langle(\nabla \widetilde{c})_{\mathrm{s}}\right\rangle$ needs to be determined from the appropriate diffusion or convective diffusion equation. For simplicity, we assume this takes the usual forms before and after linearization:

$$
\begin{aligned}
\partial c / \partial t & =D \nabla^{2} c-\mathbf{v} \cdot \nabla c \\
\mathbf{i} \omega \widetilde{c} & =D \nabla^{2} \widetilde{c}-\mathbf{v} \cdot \nabla \widetilde{c}
\end{aligned}
$$

(possibly with velocity $\mathbf{v}=0$ ), though the only requirements for the treatment here to hold are that the right-hand sides are linear and the concentration only appears there in derivatives. The assumptions and the cases covered are similar to those in a recent paper, which addresses the details ${ }^{12}$. Only the zero-frequency case is of interest here, and so the left-hand side of Eq. (5) is set equal to zero.

Determination of the steady-state limiting current is a separate problem, whose solution is found from solution of Eq. (4) with its left-hand side equal to zero. The electrode boundary condition is that the concentration at the electrode surface is zero, and the resulting current is then found from the surface flux through

$$
j_{\mathrm{L}}=n F\left\langle J_{\mathrm{s}}\right\rangle=-n F D\left\langle(\nabla c)_{\mathrm{s}}\right\rangle
$$

It is assumed that the boundary conditions at surfaces other than the electrode surface are of two types. For distant boundaries, concentration may be specified, to be the bulk concentration in the limiting current problem, or to be zero in the impedance problem. There may also be walls with zero-flux boundary conditions, for either problem. Table (I) summarizes the governing equations and boundary conditions for the two problems. The 
specification of $\widetilde{c}_{\mathrm{s}}=1$ rather than $\widetilde{c}_{\mathrm{s}}=\left(c^{\mathrm{b}} n F / R T\right) \widetilde{E}$ from Eq. (1) is for convenience; only the ratio of the surface concentration to surface flux is important (Eq. (3)).

The zero-frequency impedance and limiting current problems become identical (last column of the table) after the following respective changes of variables:

$$
\begin{aligned}
& C=1-\widetilde{c} \\
& C=c / c^{\mathrm{b}}
\end{aligned}
$$

This common problem is solved for the concentration profile, from which the average concentration gradient at the electrode $\left\langle(\nabla C)_{\mathrm{s}}\right\rangle$ is calculated. Changing back to the original variables shows that $\left\langle(\nabla C)_{\mathrm{s}}\right\rangle$ is equal to both $\left\langle(\nabla c)_{\mathrm{s}}\right\rangle / c^{\mathrm{b}}$ and $-\left\langle(\boldsymbol{\nabla} \widetilde{c})_{\mathrm{s}}\right\rangle$. Equality between these allows the product of $Z_{\mathrm{d}}$ (Eq. (3)) at zero frequency and $j_{\mathrm{L}}$ (Eq. (6)) to be simplified to $R T / n F$ :

$$
Z_{\mathrm{d}}(0)\left|j_{\mathrm{L}}\right|=R T / n F
$$

In other words, the limiting current is $R T / n F$ times the zero-frequency admittance.

The multispecies extension of this is straightforward, but the requirement to distinguish limiting reagents from others make the expressions messy. Therefore we consider only reactions with one reactant solution species and one product solution species:

$$
a \mathrm{~A}+n \mathrm{e}^{-} \rightleftharpoons b \mathrm{~B}
$$

The linearized Nernst equation becomes

$$
\widetilde{E}=\left(R T / n_{\mathrm{A}} F c_{\mathrm{A}}^{\mathrm{b}}\right) \widetilde{c}_{\mathrm{A}, \mathrm{s}}+\left(R T / n_{\mathrm{B}} F c_{\mathrm{B}}^{\mathrm{b}}\right) \widetilde{c}_{\mathrm{B}, \mathrm{s}}
$$

where $n_{i}$ is the stoichiometric coefficient of electrons divided by the stoichiometric coefficient of species $\left.i \quad \wedge n_{\mathrm{A}}=(-n) /(-a), n_{\mathrm{B}}=(-n) / b\right)$. (The quantities $n, a$ and $b$ are positive, and stoichiometric coefficients for reactants are negative.) As for the single-species case, the concentrations oscillate in phase with the potential, but unlike for the single-species equation, the two quantities $\widetilde{c}_{\mathrm{A}, \mathrm{s}}$ and $\widetilde{c}_{\mathrm{B}, \mathrm{s}}$ are not determined by this equation alone. The additional relationship between the surface ac fluxes of these two species is required to solve this problem uniquely.

Each ac flux is related to the current density by

$$
\widetilde{j}=n_{i} F\left\langle\widetilde{J}_{i, \mathrm{~s}}\right\rangle=-n_{i} F D_{i}\left\langle\left(\boldsymbol{\nabla} \widetilde{c}_{i}\right)_{\mathrm{s}}\right\rangle
$$


Dividing Eq. (11) by Eq. (12) (matching the fluxes to their respective concentrations) gives the mass transport impedance:

$$
Z_{\mathrm{d}}=\frac{\widetilde{E}}{\widetilde{j}}=\frac{R T}{n_{\mathrm{A}}^{2} F^{2} D_{\mathrm{A}} c_{\mathrm{A}}^{\mathrm{b}}} \cdot \frac{-\widetilde{c}_{\mathrm{A}, \mathrm{s}}}{\left\langle\left(\boldsymbol{\nabla} \widetilde{c}_{\mathrm{A}}\right)_{\mathrm{s}}\right\rangle}+\frac{R T}{n_{\mathrm{B}}^{2} F^{2} D_{\mathrm{B}} c_{\mathrm{B}}^{\mathrm{b}}} \cdot \frac{-\widetilde{c}_{\mathrm{B}, \mathrm{s}}}{\left\langle\left(\boldsymbol{\nabla} \widetilde{c}_{\mathrm{B}}\right)_{\mathrm{s}}\right\rangle}
$$

The anodic and cathodic limiting currents are related to the fluxes by:

$$
\begin{aligned}
& j_{\mathrm{L}, \mathrm{a}}=n_{\mathrm{B}} F\left\langle J_{\mathrm{B}, \mathrm{s}}\right\rangle=-n_{\mathrm{B}} F D_{\mathrm{B}}\left\langle\left(\boldsymbol{\nabla} c_{\mathrm{B}}\right)_{\mathrm{s}}\right\rangle \\
& j_{\mathrm{L}, \mathrm{c}}=n_{\mathrm{A}} F\left\langle J_{\mathrm{A}, \mathrm{s}}\right\rangle=-n_{\mathrm{A}} F D_{\mathrm{A}}\left\langle\left(\boldsymbol{\nabla} c_{\mathrm{A}}\right)_{\mathrm{s}}\right\rangle
\end{aligned}
$$

Similarly to the single species case, $-\widetilde{c}_{i, \mathrm{~s}} /\left\langle\left(\boldsymbol{\nabla} \widetilde{c}_{i}\right)_{\mathrm{s}}\right\rangle=1 /\left\langle\left(\nabla C_{i}\right)_{\mathrm{s}}\right\rangle=c_{i}^{\mathrm{b}} /\left\langle\left(\boldsymbol{\nabla} c_{i}\right)_{\mathrm{s}}\right\rangle$, for $i=\mathrm{A}$ or $\mathrm{B}$, which can then be related to the respective limiting currents giving:

$$
\begin{aligned}
Z_{\mathrm{d}} & =\frac{R T}{n_{\mathrm{A}}^{2} F^{2} D_{\mathrm{A}}\left\langle\left(\nabla \widetilde{c}_{\mathrm{A}}\right)_{\mathrm{s}}\right\rangle}+\frac{R T}{n_{\mathrm{B}}^{2} F^{2} D_{\mathrm{B}}\left\langle\left(\nabla \widetilde{c}_{\mathrm{B}}\right)_{\mathrm{s}}\right\rangle} \\
& =\frac{R T}{n_{\mathrm{A}}^{2} F^{2} D_{\mathrm{A}}\left(-j_{\mathrm{L}, \mathrm{c}} / n_{\mathrm{A}} F D_{\mathrm{A}}\right)}+\frac{R T}{n_{\mathrm{B}}^{2} F^{2} D_{\mathrm{B}}\left(-j_{\mathrm{L}, \mathrm{a}} / n_{\mathrm{B}} F D_{\mathrm{B}}\right)} \\
Z_{\mathrm{d}}(0) & =\frac{R T}{F\left|n_{\mathrm{A}} j_{\mathrm{L}, c}\right|}+\frac{R T}{F\left|n_{\mathrm{B}} j_{\mathrm{L}, a}\right|}=\frac{R T}{n F}\left(\frac{a}{\left|j_{\mathrm{L}, c}\right|}+\frac{b}{\left|j_{\mathrm{L}, a}\right|}\right) \\
Z_{\mathrm{d}}(0) \overline{\left|j_{\mathrm{L}}\right|} & =\frac{R T}{n F}
\end{aligned}
$$

where $\overline{\left|j_{\mathrm{L}}\right|}=\left(a\left|j_{\mathrm{L}, c}\right|^{-1}+b\left|j_{\mathrm{L}, a}\right|^{-1}\right)^{-1}$.

For the classic case of $\mathrm{O}+n \mathrm{e}^{-} \rightleftharpoons \mathrm{R}, n_{\mathrm{O}}=-n_{\mathrm{R}}=n$ and

$$
\begin{aligned}
Z_{\mathrm{d}}(0) & =\frac{R T}{n F\left|j_{\mathrm{L}, \mathrm{c}}\right|}+\frac{R T}{n F\left|j_{\mathrm{L}, \mathrm{a}}\right|} \\
Z_{\mathrm{d}}(0) \overline{\left|j_{\mathrm{L}}\right|} & =R T / n F
\end{aligned}
$$

where $\overline{\left|j_{\mathrm{L}}\right|}=\left(\left|j_{\mathrm{L}, \mathrm{c}}\right|^{-1}+\left|j_{\mathrm{L}, \mathrm{a}}\right|^{-1}\right)^{-1}$ is the harmonic mean of the anodic and cathodic limiting currents. In the special case that the diffusivities and bulk concentrations are equal, the limiting currents are equal, and the harmonic mean is half the limiting current, which leads to

$$
Z_{\mathrm{d}}(0)\left|j_{\mathrm{L}}\right|=2 R T / n F
$$

\section{RESULTS AND DISCUSSION}

In general, the mass-transport impedance involves the dc concentration at the electrode surface. It is to be emphasized that the present derivation only holds at the reversible potential, where the dc surface concentrations are equal to the bulk values for all the reaction 
species. However, the single-species mass-transport impedance considered here is the one often derived in theoretical treatments such as for the rotating disk electrode; for real systems $Z_{\mathrm{d}}$ is a sum of single-species versions for each of the diffusing species. The reversible assumption, that the ac concentrations follow the linearized Nernst equation, removes all kinetic parameters, which is strictly impossible. However, when the charge-transfer resistance is very small, the measured impedance is a good approximaton to the mass-transport impedance.

Under the reversibility assumption, and for the limiting current mass-transport problem, the mass transport of the different species are effectively uncoupled. In that case, the zerofrequency ac and steady-state dc problems follow the same governing mass-transport partial differential equation. The recognition that the boundary conditions for the ac reversible case and dc limiting current case are complementary allows the two problems to be mapped onto each other. That is, the ac concentration is zero when the dc concentration is constant, and vice versa. Likewise, the ac and dc problems both have zero-flux boundary conditions at the same locations. The governing equation must be linear, i.e., doubling the concentration gives the same equation. Also, the concentration must appear only in differentiated form, so that $C$ and $1-C$ are both solutions, in order for the transformation of Eq. (7) to hold.

In general, solutions of the convective diffusion equations for electrodes without uniform accessibility are difficult since the concentrations and fluxes vary across the electrode surface. However, for the potentiostatic impedance and limiting current problems considered here, the boundary conditions are simple: constant concentrations across the electrode surface (zero concentration for the limiting current problem, constant ac concentration for the impedance problem). The response fluxes may vary across the electrode surface, but averaging them across the surface to find the measured current density is straightforward compared to dealing with boundary conditions that vary across the electrode surface.

A few examples illustrate the range of problems that are covered. A simple example is 1D finite diffusion in a layer of thickness $d$, with boundary conditions $c_{\mathrm{s}}=0$ and $\widetilde{c}_{\mathrm{s}}=1$ at the electrode surface and $c_{d}=c^{\mathrm{b}}$ and $\widetilde{c}_{d}=0$ at the other surface. The single-species impedance is well known to be $Z_{\mathrm{d}}=\left(R T / n^{2} F^{2} c^{\mathrm{b}} \sqrt{\mathrm{i} \omega D}\right) \tanh (\sqrt{\mathrm{i} \omega / D} d)$ with zero-frequency limit $Z_{\mathrm{d}}(0)=\left(R T d / n^{2} F^{2} c^{\mathrm{b}} D\right) .{ }^{13}$ The limiting current is $j_{\mathrm{L}}=-n F D c^{\mathrm{b}} / d$, and the product is $R T / n F$ as predicted by Eq. (9).

The dimensionless zero-frequency mass-transport impedance for the rotating disk elec- 
trode has been given ${ }^{7,14}$ as $-1 / \theta^{\prime}(0)=\Gamma(4 / 3)$ where the prime denotes the derivative relative to $z / \delta, z$ is the normal distance from the electrode, and $\delta=(3 D / a \nu)^{1 / 3}(\nu / \Omega)^{1 / 2}$. (Here $\Omega$ is the rotation rate, $\nu$ is the kinematic viscosity, and $a=0.510233$ is a dimensionless parameter.) Converting to a derivative with respect to $z$ gives $-\widetilde{c}_{\mathrm{s}} /\left(\boldsymbol{\nabla} \widetilde{c}_{\mathrm{s}}\right)=\Gamma(4 / 3) \delta$ at zero frequency and therefore $Z_{\mathrm{d}}(0)=\Gamma(4 / 3) \delta R T / n^{2} F^{2} D c^{\mathrm{b}}$. This is in the so-called infinite Schmidt number approximation, for which the corresponding limiting current is given by the Levich formula, $j_{\mathrm{L}}=0.62 n F D^{2 / 3} \nu^{-1 / 6} \Omega^{1 / 2} c^{\mathrm{b}}$, where the exact form for 0.62 is $\Gamma(4 / 3)^{-1}(a / 3)^{1 / 3}$. The product again simplifies to $R T / n F$.

The inlaid disk electrode provides a more interesting case, since it is not uniformly accessible. The limiting current density was shown by Saito ${ }^{15}$ to be $j_{\mathrm{L}}=4 n F D c^{\mathrm{b}} / \pi r_{\mathrm{e}}$, where $r_{\mathrm{e}}$ is the disk radius. The classic impedance derivations for this system were by Fleischmann and Pons ${ }^{16,17}$, who recognized that the boundary condition should be uniform concentration across the surface, but for mathematical simplicity used a uniform flux and then averaged the calculated surface concentrations ${ }^{18}$. This was remedied by later workers ${ }^{19,20}$. Michel et $\mathrm{al}^{20}$ used accurate numerical simulations for the general case of the reaction $\mathrm{O}+n \mathrm{e} \rightleftarrows$ $\mathrm{R}$ with Butler-Volmer kinetics, and showed that for large standard rate constants the ac concentration was uniform across the surface. They showed numerically for fast rates at the reversible potential and with equal diffusivities and bulk concentrations that the mass transport part of the impedance at zero frequency approached $\pi / 2$ in some dimensionless units. Converting to real units gives $Z_{\mathrm{d}}(0)=R T \pi r_{\mathrm{e}} / 2 n^{2} F^{2} D c^{\mathrm{b}} \quad$ (Their $R_{\mathrm{d}}$ is in ohms and needs to be multiplied by the area $\pi r_{\mathrm{e}}^{2}$.) The product of $Z_{\mathrm{d}}(0)$ with Saito's $j_{\mathrm{L}}$ gives $2 R T / n F$, in agreement with Eq. (22).

\section{SUMMARY}

The conditions for the relationship between the zero-frequency reversible mass-transport impedance and the limiting current to hold are established by finding an equivalence between their respective mass-transport governing equations and boundary conditions. 


\begin{tabular}{|l|l|l|l|}
\cline { 2 - 4 } \multicolumn{1}{c|}{} & impedance & limiting current & common \\
\hline governing equation & $0=D \nabla^{2} \widetilde{c}-\mathbf{v} \cdot \nabla \widetilde{c}$ & $0=D \nabla^{2} c-\mathbf{v} \cdot \nabla c$ & $0=D \nabla^{2} C-\mathbf{v} \cdot \boldsymbol{\nabla} C$ \\
electrode surface & $\widetilde{c}_{\mathrm{s}}=1$ & $c_{\mathrm{s}}=0$ & $C_{\mathrm{s}}=0$ \\
wall & $\nabla \widetilde{c}_{\mathrm{s}}=0$ & $\nabla c_{\mathrm{S}}=0$ & $\nabla C_{\mathrm{s}}=0$ \\
far boundary & $\widetilde{c}_{\mathrm{s}}=0$ & $c_{\mathrm{s}}=c^{\mathrm{b}}$ & $C_{\mathrm{s}}=1$ \\
\hline
\end{tabular}

TABLE I. Governing and boundary conditions for limiting current and impedance problems

\section{ACKNOWLEDGMENTS}

I thank the Natural Sciences and Engineering Research Council of Canada (NSERC) for financial support of this research.

\section{REFERENCES}

${ }^{1}$ M Sluyters-Rehbach and J.H. Sluyters p 1 in A.J. Bard, ed., Electroanalytical Chemistry, vol 4, Marcel Dekker, New York (1970).

${ }^{2}$ D. Schuhmann, Electrochim. Acta, 35, 1527 (1990).

${ }^{3}$ A.J. Bard and L.R. Faulkner, Electrochemical Methods, Fundamentals and Applications, 2nd Ed., Wiley, 2001, p. 34.

${ }^{4}$ T. Jacobsen and K. West, Electrochim. Acta, 40, 255 (1995).

${ }^{5}$ R.V. Homsy, J. Newman, J. Electrochem. Soc., 121, 521 (1974).

${ }^{6}$ A. Molina, J. Gonzalez, M.C. Henstridge, R.G. Compton, J. Phys. Chem. C, 115, 4054 (2011).

${ }^{7}$ D.A. Scherson, J. Newman, J. Electrochem. Soc., 127, 110 (1980).

${ }^{8}$ A. Bezegh, J. Janata, J. Electroanal. Chem., 215, 139 (1986).

${ }^{9}$ R.G. Compton, J. Winkler, J. Phys. Chem., 99, 5029 (1995).

${ }^{10}$ J.A. Alden, R.G. Compton, Electroanalysis, 8, 30 (1996).

${ }^{11}$ M.T.T. Tran, B. Tribollet, V. Vivier, M.E. Orazem, Russ. J. Electrochem., 53, 932 (2017).

${ }^{12}$ D. A. Harrington, Electrochim. Acta, 152, 308 (2015).

${ }^{13}$ A. Lasia, Electrochemical Impedance Spectroscopy and its Applications, p. 104, Springer, New York (2014).

${ }^{14}$ B. Tribollet and J. Newman, J. Electrochem. Soc., 130, 822 (1983). 
${ }^{15}$ Y. Saito, Rev. Polarography, 15, 177 (1968).

${ }^{16}$ M. Fleischmann and S. Pons, J. Electroanal. Chem., 250, 277 (1988).

${ }^{17}$ M. Fleischmann and S. Pons, J. Daschbach, J. Electroanal. Chem., 317, 1 (1991).

${ }^{18}$ M. Fleischmann and S. Pons, J. Electroanal. Chem., 250, 269 (1988).

${ }^{19}$ R. Ferrigno and H. H. Girault, J. Electroanal. Chem., 492, 1 (2000).

${ }^{20}$ R. Michel, C. Montella, C. Verdier and J.-P. Diard, Electrochim. Acta, 55, 6263 (2010). 